\title{
DUAL ACCELEROMETER USAGE STRATEGY FOR ONBOARD SPACE NAVIGATION
}

\author{
Renato Zanetti* and Chris D'Souza ${ }^{\dagger}$
}

\begin{abstract}
This work introduces a dual accelerometer usage strategy for onboard space navigation. In the proposed algorithm the accelerometer is used to propagate the state when its value exceeds a threshold and it is used to estimate its errors otherwise. Numerical examples and comparison to other accelerometer usage schemes are presented to validate the proposed approach.
\end{abstract}

\section{INTRODUCTION}

In the presence of significant and consistent non-gravitational accelerations, accelerometer measurements are often used in lieu of analytical expressions to propagate the state of aerospace vehicles in model-based estimation algorithms such as the Kalman filter [1, 2]. In space applications, accelerometers are often used to propagate the state of the vehicle through a translational maneuver, or "burn". At all other times non-gravitational forces are usually much smaller than the accuracy of most commercially available accelerometers, therefore space vehicles commonly threshold the acceleromter, i.e. they use it only when the measurements are above a predetermined value [3]. The Space Shuttle rendezvous and proximity operations program (RPOP), for example, employs this strategy [4].

Rarely accelerometers are used as external measurements to update the state of the vehicle. One example of this approach is Ref. [5] in which accelerometers are used in conjunction with a filter bank for Mars entry navigation. Under this type of implementation, a model of the non-gravitational forces is required. When on a launch pad a vehicle is stationary with respect to Earth and the predicted accelerometer measurement is very easily obtained. Pre-launch operations therefore commonly employ accelerometer measurements to update the estimate of its repeatable errors, such as biases, misalignments, and scale factors.

Accelerometers usually have two types of repeatable biases. A constant bias (sometimes represented as a first order Markov process [6] with very large time constant of the order of 24 hours) and a faster changing Markov process with time constant of around one hour. Velocity random walk (VRW) also corrupts the measurement, but this source of error is not estimable because it is white acceleration noise. In the standard accelerometer usage by space vehicles the accelerometer errors are estimated from external measurements

* Senior Member of the Technical Staff, Vehicle Dynamics and Controls, The Charles Stark Draper Laboratory, 17629 El Camino Real, Suite 470, Houston, Texas, 77058. rzanetti@ draper.com

${ }^{\dagger}$ GN\&C Autonomous Flight Systems Engineer, Aeroscience and Flight Mechanics Division, NASA Johnson Space Center EG6, 2101 NASA Parkway, Houston, Texas, 77058. chris.dsouza@nasa.gov 
through their correlation to other states. This correlation is built during maneuvers at which times the accelerometer measurement is incorporated into the filter dynamics. In between maneuvers the accelerometer measurement is not used at all, therefore the estimate of the one-hour Markov bias degrades, which results in a worst navigation performance during the next maneuver. For vehicles with small thrusters and maneuvers far apart, this approach is often not sufficient to meet mission goals. A common practice for these vehicles is to use a simple averaging scheme to estimate the accelerometer bias prior to maneuvers. This average is carried outside the navigation filter, therefore no correlation between the filter error and the accelerometer bias estimation error is taken into account when formulating a navigation solution.

This work introduces a dual accelerometer usage strategy for onboard space navigation. In the proposed algorithm the accelerometer is used to propagate the state when its value exceeds a threshold and it is used to estimate its errors otherwise. The paper is organized as follows: accelerometer thresholding is first introduced, the navigation algorithm is then presented and validated with numerical simulation, finally conclusions are drawn.

\section{ACCELEROMETER THRESHOLDING}

This section presents a simple example to show the need of accelerometer thresholding in spacecraft navigation. Outside of thrust, drag is usually the biggest non-gravitational acceleration source in low Earth orbit (LEO). The international space station (ISS) is used as an example to quantify the contribution of drag. The ISS is a large structure placed at an altitude of $400 \mathrm{~km}$ and is subject to high drag. A 2008 analysis from the European Space Agency* finds that the ISS coefficient of drag is 2.07, the frontal area between 700 and $2300 \mathrm{~m}^{2}$ depending on the configuration, and the yearly average air density 3.98 $10^{-12} \mathrm{~kg} / \mathrm{m}^{3}$. Assuming a configuration with frontal area $1000 \mathrm{~m}^{2}$ the drag acting on the ISS is $0.25 \mathrm{~N}$. The mass of ISS was $2.510^{5} \mathrm{~kg}$ in 2008 , resulting in a drag acceleration of $10^{-6} \mathrm{~m} / \mathrm{s}^{2}$ or $0.1 \mu \mathrm{g}$.

A relatively good accelerometer can have a one hour markov with steady-state standard deviation $\sigma_{s s}=10 \mu \mathrm{g}$. For each accelerometer's axis, the markov bias $b_{a}$ evolves as

$$
\dot{b}_{a}=-\frac{1}{\tau} b_{a}+\nu
$$

where $\tau=3600 \mathrm{~s}$ and $\nu$ is a zero-mean white process with spectral density $S_{\nu}=\sigma_{s s}^{2} /(2 \tau)$. In order to establish to which accuracy this markov bias can be estimated, we assume a continuous Kalman filter, we also assume no non-gravitational forces acting on the vehicle, therefore the accelerometer measurement is

$$
y=b_{a}+\eta
$$

for each axis. The velocity random walk $\eta$ is a zero-mean white process, a spectral density $S_{\eta}=(10 \mu g \sqrt{s})^{2}$ is assumed, which is a relatively good value. The estimate of the markov

\footnotetext{
*http://www.esa.int/esaMI/Space_In_Bytes/SEM7FXJ26DF_0.html
} 
bias evolves as

$$
\dot{\hat{b}}_{a}=-\frac{1}{\tau} \hat{b}_{a}+K\left(y-\hat{b}_{a}\right),
$$

where $K=P / S_{\eta}$ and $P$ is the estimation error variance evolving as

$$
\dot{P}=-\frac{2}{\tau} P+\frac{2}{\tau} \sigma_{s s}^{2}-P^{2} / S_{\eta}
$$

After some algebra it follows that the steady-state value of the estimation error standard deviation is $\sqrt{P_{s s}}=1.5 \mu \mathrm{g}$. Even under the optimistic assumptions of this example the estimation error of the markov bias is 15 times bigger than drag. When using the compensated accelerometer measurement to propagate the state an additional error source is the VRW. During non-thrusting phases it is therefore much more accurate not to use the accelerometer to propagate the state but to use a simple drag model.

\section{DUAL ACCELEROMETER USAGE STRATEGY}

In all spacecraft missions the authors are aware of accelerometers are thresholded during orbital coast flight (sometimes this operation is referred to as accelerometer gating). In the majority of this mission the accelerometer bias is estimated to improve the navigation solution. The estimation occurs in either of two ways. A common solution is to include the accelerometer bias as a state in the filter and to estimate it through external measurements and the correlations built during maneuvers. The issue with this approach is that when maneuvers occur far apart the estimate degrades in-between maneuvers, often resulting in a poor knowledge of the bias when the following maneuver occurs. The usual solution to this problem is to have an external estimator of the accelerometer bias. While this second solution usually produces good results, it completely ignores the inevitable correlation between the states in the filter and the accelerometer bias estimate. To optimally account for this correlation we propose an integrated filter that uses the estimate of the bias during maneuvers and estimates it during coast flight.

Accelerometer measurements fed to the navigation filter are usually integrated accelerations over the last time step and they are compensated for sculling errors. Sculling compensation means that the effects of the rotation of the vehicle during the time step are compensated and the measurement $\Delta \tilde{\mathbf{v}}_{k}^{b}$ is an inertial change in velocity between times $t_{k-1}$ and $t_{k}$ coordinatized in the body-fixed frame at time $t_{k}$. When the filter is called it first propagates the position (r), velocity ( $\mathbf{v})$, and accelerometer bias (b) to the current IMU time $t_{k}$ as

$$
\begin{aligned}
\frac{d}{d t} \hat{\mathbf{r}}^{i} & =\hat{\mathbf{v}}^{i} \\
\frac{d}{d t} \hat{\mathbf{v}}^{i} & =\hat{\mathbf{g}}^{i}+\hat{\mathbf{a}}^{i} \\
\frac{d}{d t} \hat{\mathbf{b}}^{b} & =-\frac{1}{\tau} \mathbf{b}_{a}^{b} .
\end{aligned}
$$

When the accelerometer measurement is above a threshold the estimated non-gravitational acceleration $\hat{\mathbf{a}}^{i}$ is obtained from the accelerometer measurement $\hat{\mathbf{a}}^{i}=\mathbf{T}_{b}^{i}\left(t_{k}\right)\left(\Delta \tilde{\mathbf{v}}_{k}^{b} / \Delta t-\right.$ 
$\left.\hat{\mathbf{b}}^{b}\right)$. When the measurement is below the threshold $\hat{\mathbf{a}}^{i}$ is either zero or a model of drag, depending on the application. Matrix $\mathbf{T}_{b}^{i}\left(t_{k}\right)$ is the coordinate transformation from the body-fixed frame at time $t_{k}$ to the inertial frame. Other states such as attitude can easily be incorporated in the filter.

When the accelerometer is included during propagation the state is only updated with external measurements. When the accelerometer is thresholded an estimate of the measurement $\Delta \hat{\mathbf{v}}_{k}^{b}$ is formed. This estimate is given by

$$
\Delta \hat{\mathbf{v}}_{k}^{b}=\int_{t_{k-1}}^{t_{k}}\left(\mathbf{b}_{a}^{b}+\mathbf{d}^{b}\right) d t=\tau\left(e^{\Delta t / \tau}-1\right) \mathbf{b}\left(t_{k}\right)+\int_{t_{k-1}}^{t_{k}} \mathbf{d}^{b} d t \simeq \Delta t\left(\mathbf{b}\left(t_{k}\right)+\mathbf{d}^{b}\right),
$$

The measurement mapping matrix for the accelerometer measurement, $\mathbf{H}_{a}$ is a $3 \times n$ matrix, where $n$ is the number of states in the filter. Matrix $\mathbf{H}_{a}$ has zeros everywhere except for an identity matrix times $\Delta t$ at the $3 \times 3$ block corresponding the accelerometer bias state. The Kalman gain is calculated as usual

$$
\mathbf{K}_{k}=\mathbf{P}_{k}^{-} \mathbf{H}_{a}^{\mathrm{T}}\left(\mathbf{H}_{a} \mathbf{P}_{k}^{-} \mathbf{H}^{\mathrm{T}}+\mathbf{R}_{a}\right)^{-1},
$$

where $\mathbf{P}_{k}^{-}$is the a priori estimation error covariance matrix and $\mathbf{R}_{a}$ is the accelerometer noise covariance. The accelerometer white noise is expressed in terms of a velocity random walk with an associated spectral density $\mathbf{S}_{a}$ whose units are the square of $m / s / \sqrt{s}$ or, more frequently, $\mu g \sqrt{s}$. Therefore the covariance of the noise over an IMU step $\Delta t$ is given by

$$
\mathbf{R}_{a}=\mathbf{S}_{a} \Delta t
$$

It is not desirable to update all the states, only the accelerometer bias should be updated. Therefore a consider gain $\mathbf{K}_{k}^{*}$ is formed by zeroing all the rows of $\mathbf{K}_{k}$ corresponding to the other states. Since the new gain is not optimal the Joseph's formula is used to obtain the a posteriori covariance

$$
\mathbf{P}_{k}^{+}=\left(\mathbf{I}_{n \times n}-\mathbf{K}_{k}^{*} \mathbf{H}_{a}\right) \mathbf{P}_{k}^{-}\left(\mathbf{I}_{n \times n}-\mathbf{K}_{k}^{*} \mathbf{H}_{a}\right)^{\mathrm{T}}+\mathbf{K}_{k}^{*} \mathbf{R}_{a}\left(\mathbf{K}_{k}^{*}\right)^{\mathrm{T}}
$$

The state is updated as

$$
\hat{\mathbf{x}}_{k}^{+}=\hat{\mathbf{x}}_{k}^{-}+\mathbf{K}_{k}^{*}\left(\Delta \tilde{\mathbf{v}}_{k}^{b}-\Delta \hat{\mathbf{v}}_{k}^{b}\right) .
$$

The choice of the threshold value is usually driven by the thruster size, the accelerometer accuracy, and engineering judgement. A good rule of thumbs is to threshold the accelerometer when the measurement is below the $3 \sigma$ value of the sum of the bias and noise, assumed independent

$$
\left(\Delta \tilde{\mathbf{v}}_{k}^{b}\right)^{\mathrm{T}} \Delta \tilde{\mathbf{v}}_{k}^{b}<9\left(\operatorname{trace} \mathbf{B}_{a} \Delta t^{2}+\operatorname{trace} \mathbf{S}_{a} \Delta t\right),
$$

where $\mathbf{B}_{a}$ is the accelerometer bias covariance from the IMU specifications. If $\Delta \tilde{\mathbf{v}}_{k}^{b}$ is compensated with the estimate of the bias, $\mathbf{B}_{a}$ is replaced with the accuracy of the bias estimate. It is also possible to choose a non-constant threshold

$$
\left(\Delta \tilde{\mathbf{v}}_{k}^{b}-\hat{\mathbf{b}}^{b} \Delta t\right)^{\mathrm{T}}\left(\Delta \tilde{\mathbf{v}}_{k}^{b}-\hat{\mathbf{b}}^{b} \Delta t\right)<9\left(\operatorname{trace} \mathbf{P}_{a a}^{-} \Delta t^{2}+\operatorname{trace} \mathbf{S}_{a} \Delta t\right)
$$


where $\mathbf{P}_{a a}^{-}$is the $3 \times 3$ portion of the covariance corresponding to the accelerometer bias state. Spacecrafts usually operate at low angular rates, but in case of high rates the term $\hat{\mathbf{b}}^{b} \Delta t$ in Eq. (14) needs to be replaced by

$$
\int_{t_{k-1}}^{t_{k}} \mathbf{T}_{b(\tau)}^{b\left(t_{k}\right)} \mathbf{b}^{b}(\tau) d \tau
$$

where $\mathbf{T}_{b(\tau)}^{b\left(t_{k}\right)}$ is the transformation matrix that takes the vehicle's body frame at time $\tau$ into the body frame at time $t_{k}$.

This section assumes that the entire accelerometer measurement is either applied or thresholded, it is also possible to develop similar algorithms by considering the measurement from each axis independently.

\section{NUMERICAL RESULTS}

An orbital rendezvous is used as an example. The target spacecraft is placed on a circular $400 \mathrm{~km}$ orbit. The chaser vehicle is placed in a circular orbit $4 \mathrm{~km}$ below the target and $14 \mathrm{~km}$ behind. At a downrange of $13.2 \mathrm{~km}$ an altitude rase maneuver is commanded. This maneuver is a 135 degree lambert targeting taking the chaser to $1.4 \mathrm{~km}$ below and $4.3 \mathrm{~km}$ behind the target. A clean up maneuver is performed half the way during this transfer. A circularization maneuver is performed once the end of the transfer (2030s). A third maneuver is performed that will take the chaser $500 \mathrm{~m}$ below the target with zero relative velocity. Figure 1 shows the relative trajectory. The origin is the location of the target, while the line represent the relative position in time. The $y$-axis is the altitude, while the $x$-axis is downrange.

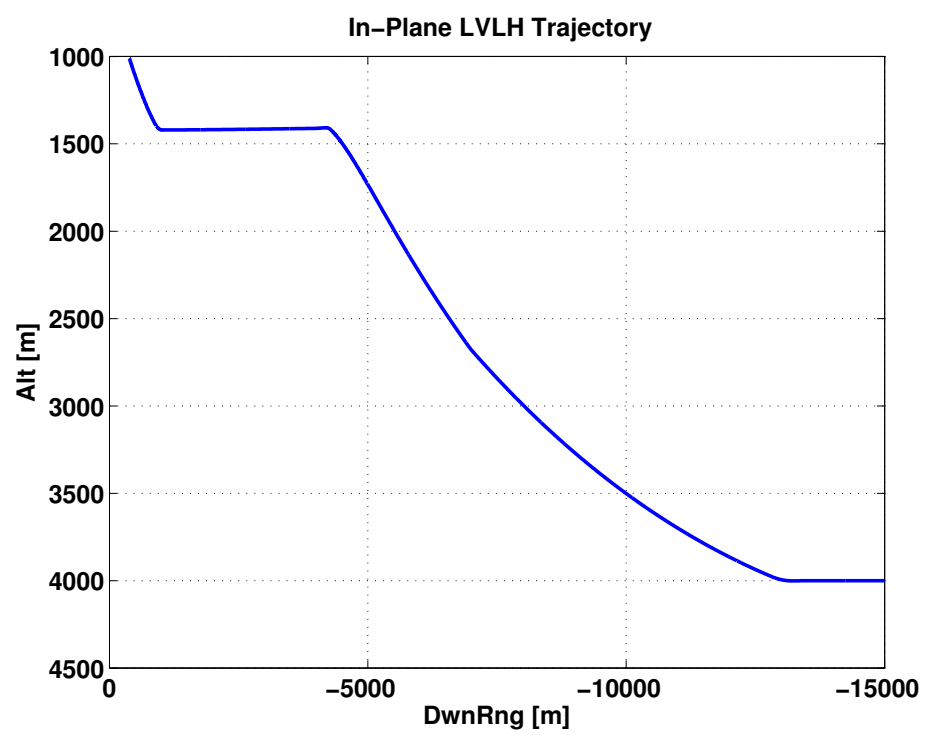

Figure 1. Relative In-Plane LVLH Trajectory 
The simulated true environment contains a 9 by 9 gravity model and atmospheric drag. The filter is a nine state filter with relative position, velocity, and accelerometer bias. The propagation includes $\mathrm{J} 2$ effects and no drag. The chaser vehicle has a $20 \mathrm{~N}$ thruster and a mass of $1000 \mathrm{~kg}$. The accelerometer errors are given in Table 1.

\begin{tabular}{ccc}
\hline \hline Error Type & $1 \sigma$ Error & Units \\
\hline Time Constant & 3600 & $\mathrm{~s}$ \\
Markov Bias & 100 & $\mu \mathrm{g}$ \\
Velocity Random Walk & 100 & $\mu \mathrm{g} \sqrt{\mathrm{s}}$ \\
\hline \hline
\end{tabular}

Table 1. Accelerometer Model Error Parameters

Together with the accelerometer there is a long range radar whose errors are shown in Table 2. The radar provides range to the target as well as azimuth and elevation to it.

\begin{tabular}{ccc}
\hline \hline Error Type & $1 \sigma$ Error & Units \\
\hline Range Error & 5 & $\mathrm{~m}$ \\
Angles Errors & 0.5 & $\mathrm{deg}$ \\
\hline \hline
\end{tabular}

Table 2. Radar Model Error Parameters

The initial filter covariance is diagonal with entries as shown in Table 3, while the velocity process noise covariance is $10^{-6} \mathrm{~m}^{2} / \mathrm{s}^{3}$.

\begin{tabular}{ccc}
\hline \hline Error Type & $1 \sigma$ Error & Units \\
\hline Relative Position & 10 & $\mathrm{~m}$ \\
Relative Velocity & 0.1 & $\mathrm{~m} / \mathrm{s}$ \\
Accelerometer Bias & 100 & $\mu \mathrm{g}$ \\
\hline \hline
\end{tabular}

Table 3. Initial Estimation Errors

Figs. 2 and 3 show the filter's performance without thresholding the accelerometer (i.e. the accelerometer measurement is always used to propagate the vehicle state). The lines show the estimation error standard deviation. Figs. 4 and 5 show the filter's performance with thresholding the accelerometer. Figs. 6 and 7 show the filter's performance when the accelerometer bias is estimated from the accelerometer measurements outside maneuvers. Figs. 8 and 9 show a direct comparison of the three methods. It can be seen that the new algorithm outperforms the simple thresholding scheme during firing of the thrusters.

\section{CONCLUSIONS}

This papers presents a dual accelerometer usage in an orbital Kalman filter. The accelerometer is both used to propagate position and velocity during maneuvers and to update 


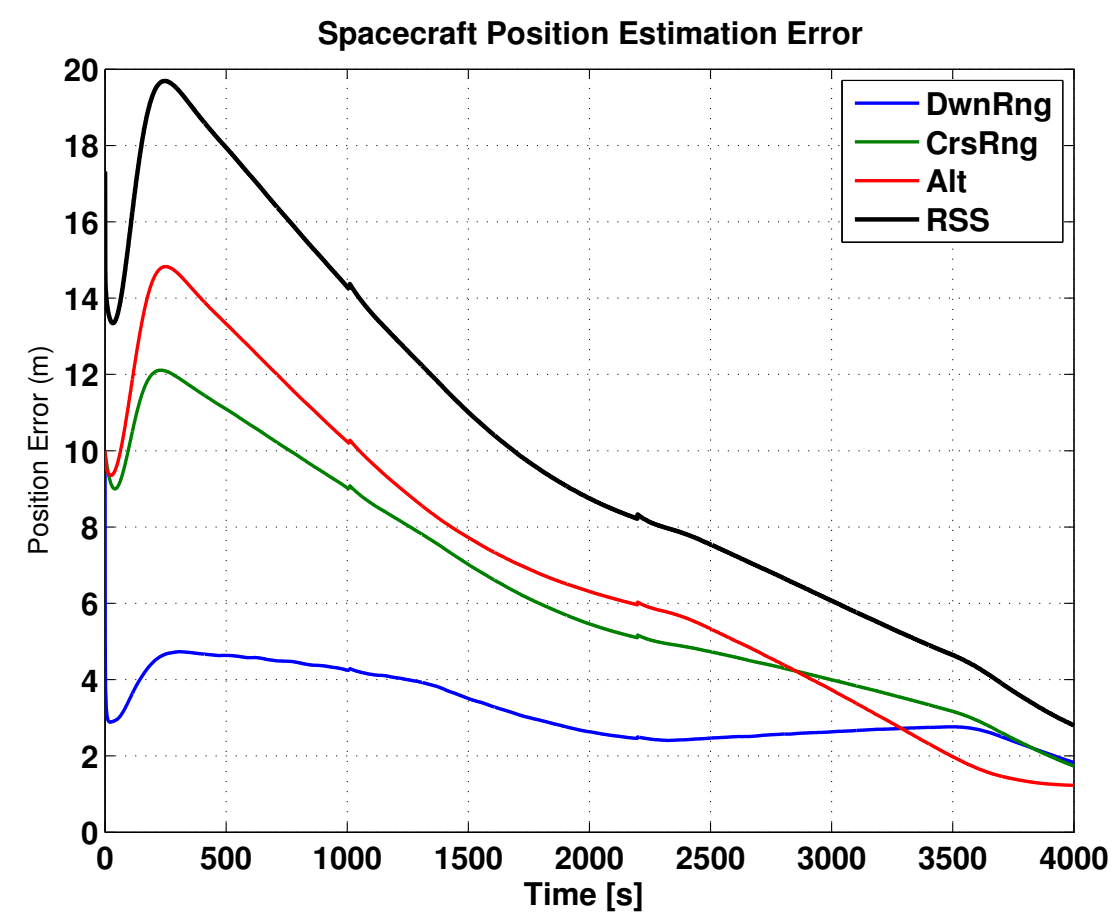

Figure 2. Position Estimation Error Without Thresholding

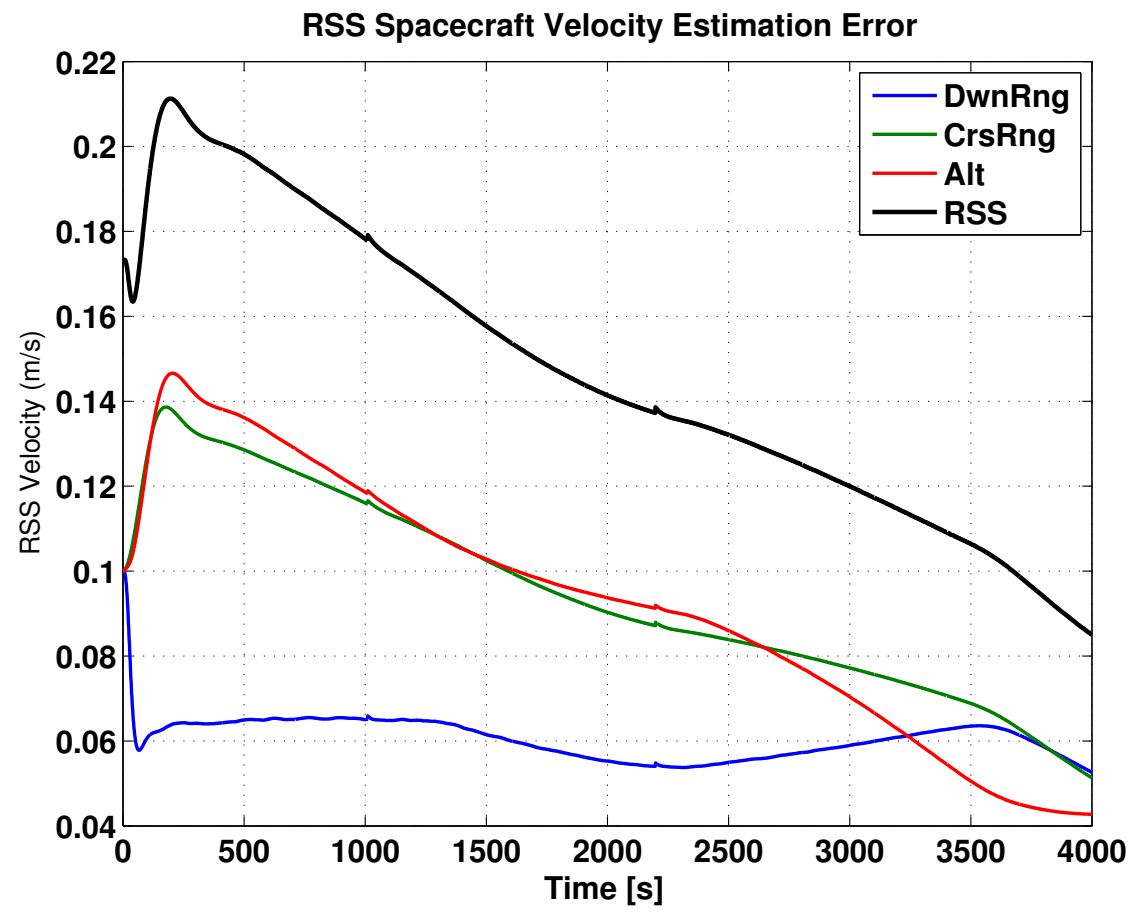

Figure 3. Velocity Estimation Error Without Thresholding 


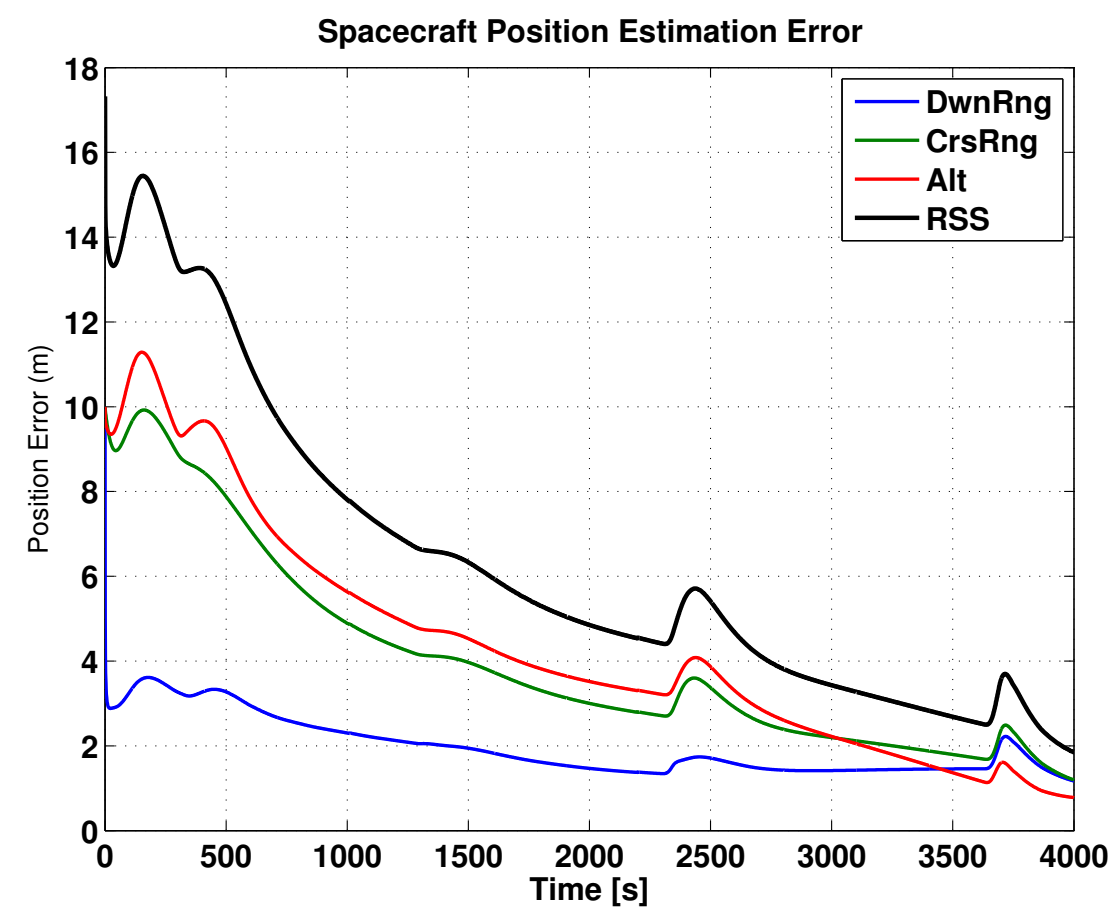

Figure 4. Position Estimation Error With Thresholding

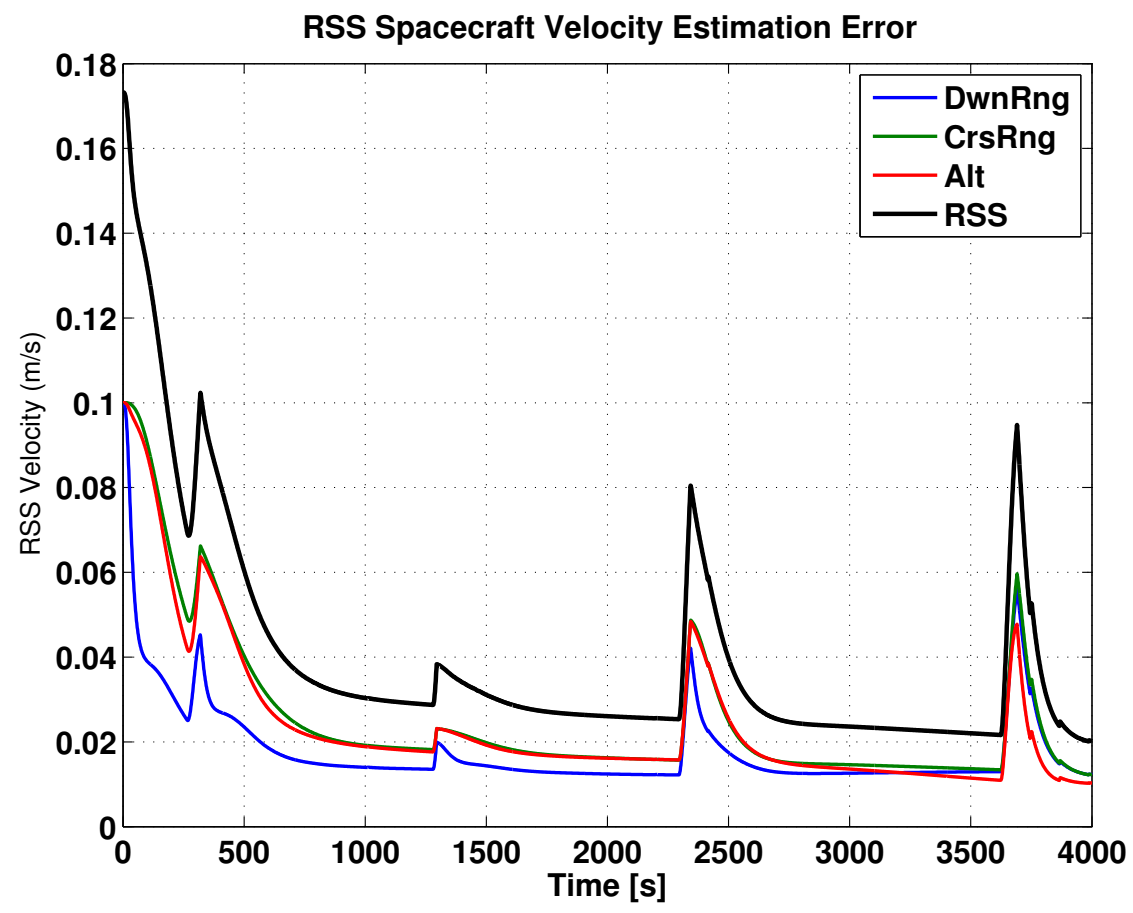

Figure 5. Velocity Estimation Error With Thresholding 


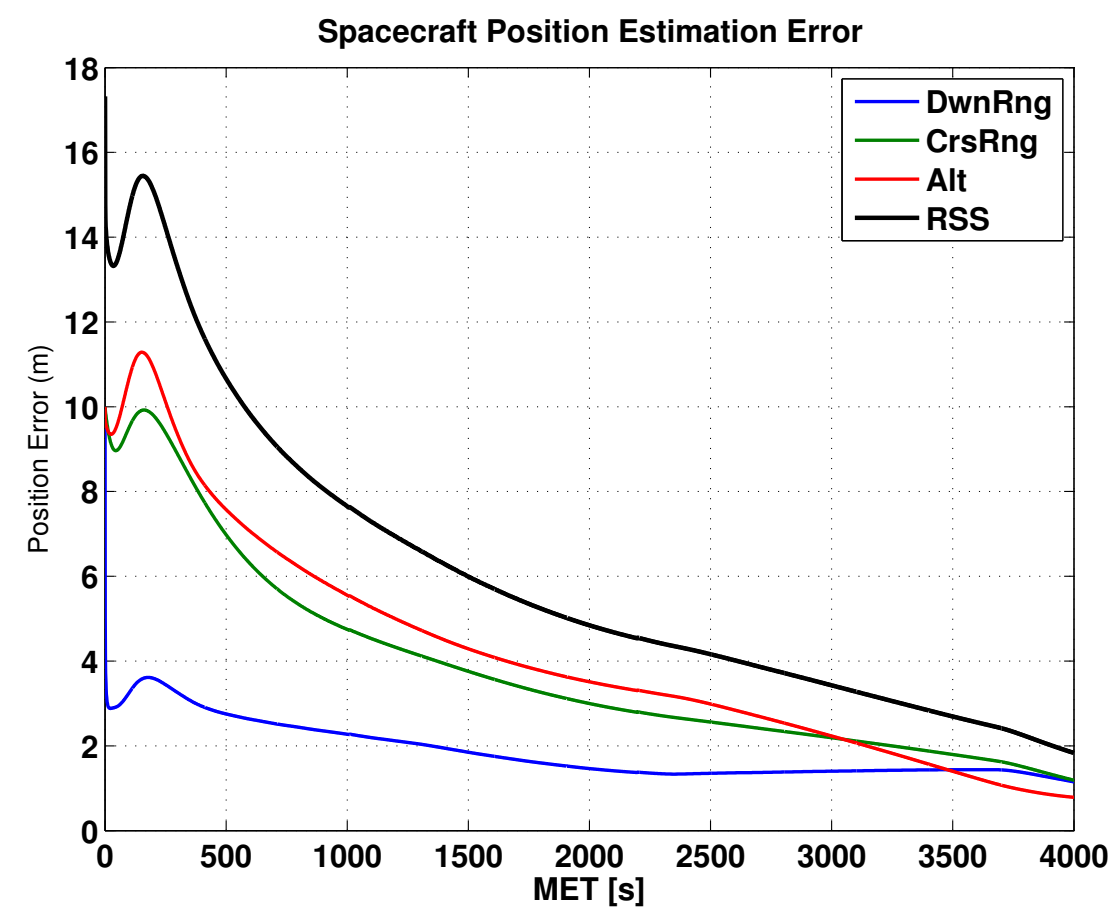

Figure 6. Position Estimation Error New Algorithm

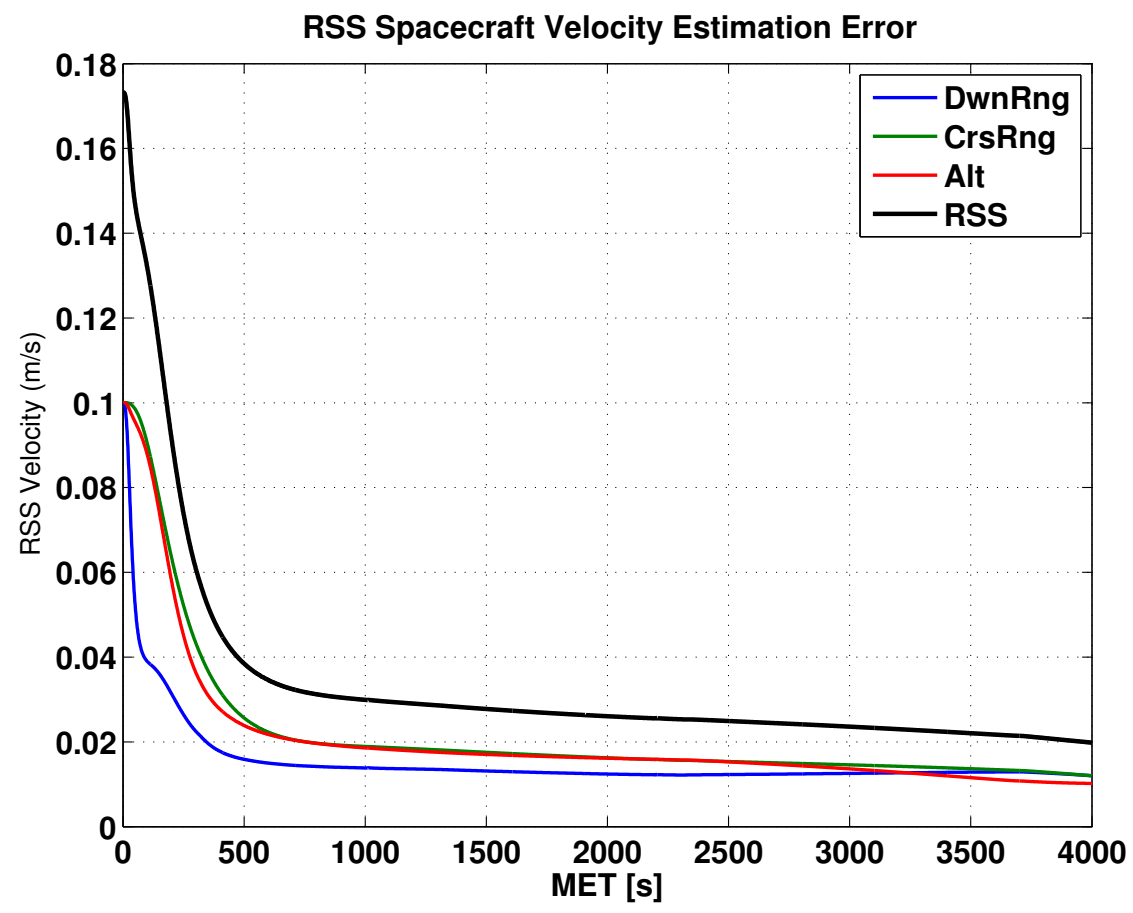

Figure 7. Velocity Estimation Error New Algorithm 


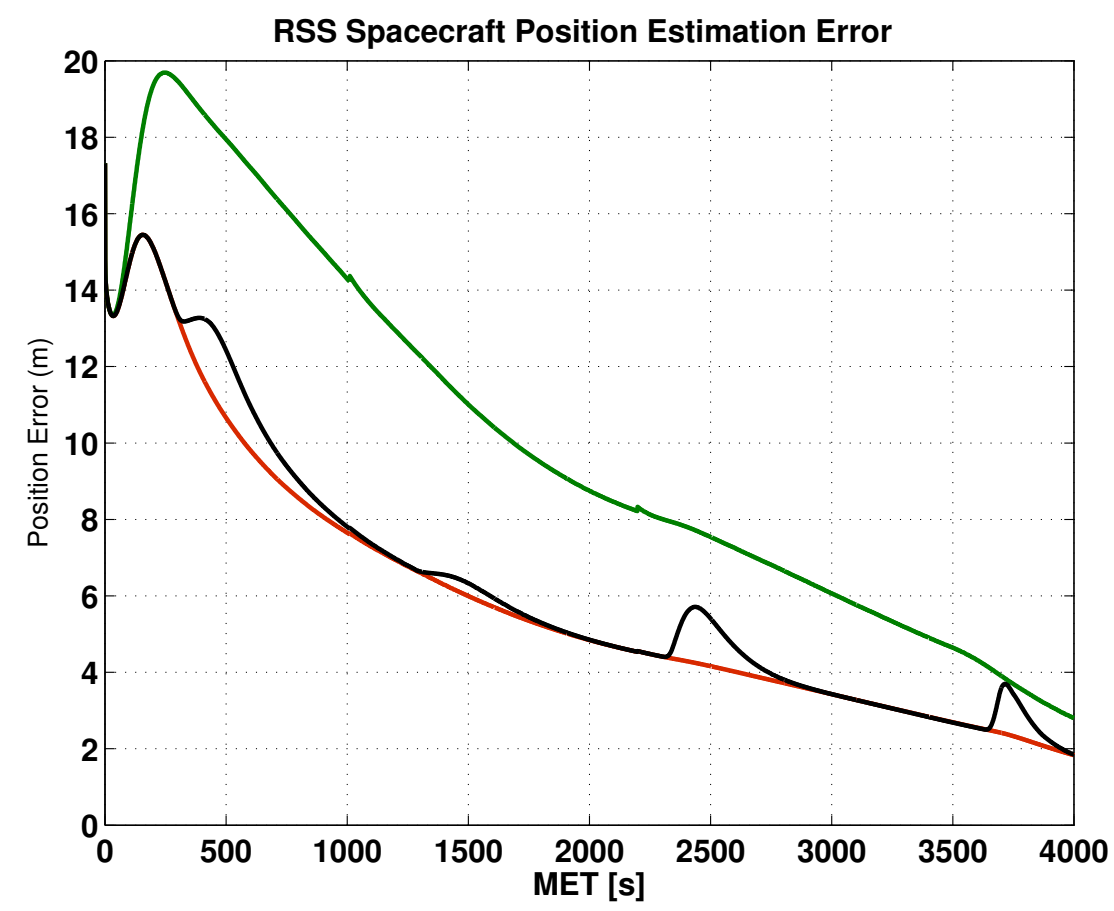

Figure 8 Comparison of Position Estimation Error, green line shows no threshold, black line with threshold and red line is the new algorithm

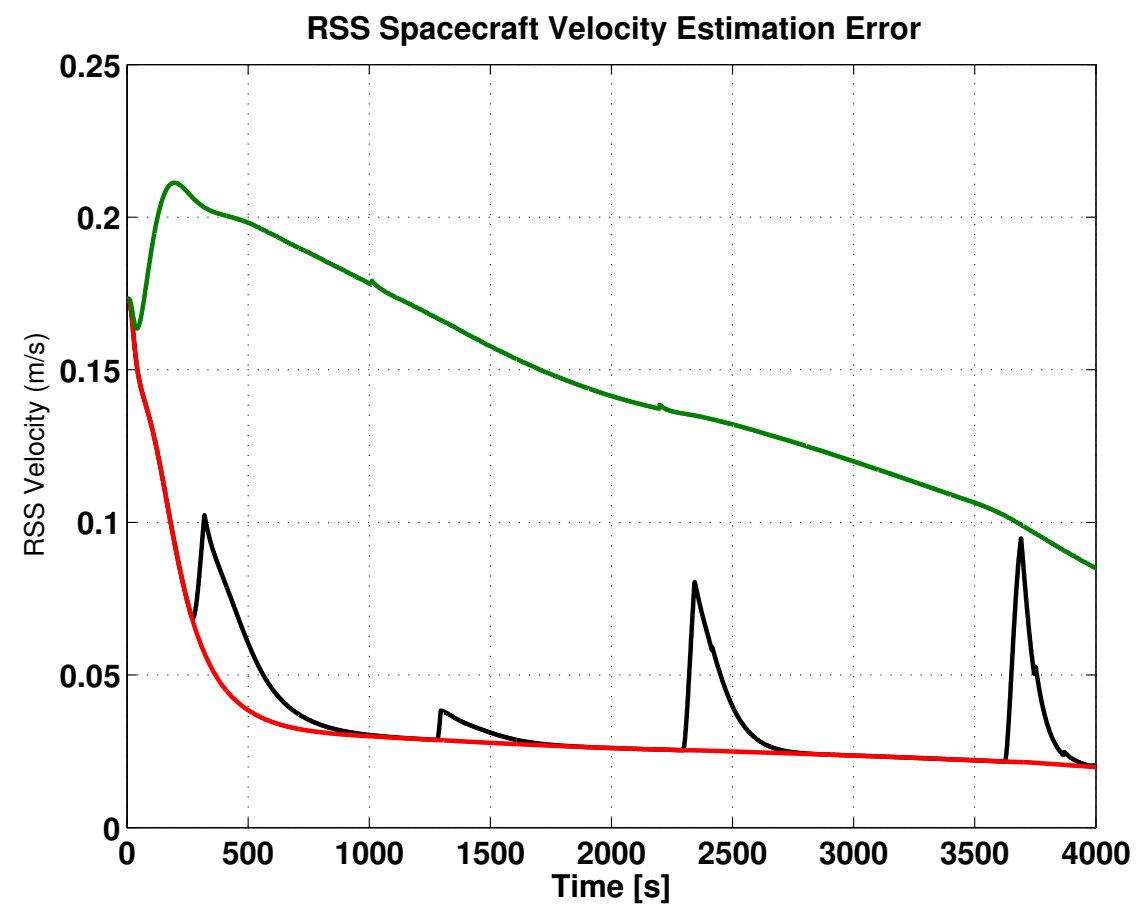

Figure 9 Comparison of Velocity Estimation Error, green line shows no threshold, black line with threshold and red line is the new algorithm 
the state outside of maneuvers. The advantage of this approach is its superior performance than simply thresholding the accelerometer. The correlation between accelerometer bias and position and velocity during the maneuvers is not sufficient to adequately estimate the bias during coast flight. Therefore the estimate of the bias degrades outside of maneuvers adding considerable uncertainty during subsequent maneuvers. A common solution to this problem is to estimate the accelerometer bias outside of the navigation filter just prior to a maneuver is performed. A simple averaging scheme is often used, but a Kalman filter is also a possibility. The advantage of the proposed scheme over a separate bias estimator is that a single estimator is globally optimal because it accounts for all the correlations.

\section{REFERENCES}

[1] Kalman, R. E., “A New Approach to Linear Filtering and Prediction Problems," Journal of Basic Engineering, Vol. 82, No. Series D, March 1960, pp. 35-45.

[2] Kalman, R. E. and Bucy, R. S., "New Results in Linear Filtering and Prediction," Journal of Basic Engineering, Vol. 83, No. Series D, March 1961, pp. 95-108.

[3] Bishop, R. H., Crain, T. P., and Azimov, D. M., "Sensitivity of Mars Entry Navigation Errors to Sensed Accelerometer Threshold," Proceedings of the AIAA Guidance, Navigation, and Control Conference, Austin, TX, 11-14 August 2003, AIAA-2003-5749.

[4] Clark, F. D., Spehar, P. T., Brazzel, J. P., and Hinkel, H. D., "Laser-Based Relative Navigation and Guidance for Space Shuttle Proximity Operations," Proceedings of the 26th Annual AAS Guidance and Control Conference, Breckenridge, CO, February 5-9 2003, pp. 521-529.

[5] Zanetti, R. and Bishop, R. H., “Adaptive Entry Navigation Using Inertial Measurements," Proceedings of the 2007 AAS/AIAA Spaceflight Mechanics Meeting held January 28 - Febraury 1, 2007, Sedona, Arizona, Vol. 127 of Advances in the Astronautical Sciences, 2007, pp. 457-470, AAS 07-129.

[6] Brown, R. G. and Hwang, P. Y., Introduction To Random Signals And Applied Kalman Filtering, John Wiley and Sons, 3rd ed., 1997. 